\title{
A Note on Congruent Numbers
}

\author{
By Ronald Alter and Thaddeus B. Curtz
}

\begin{abstract}
An integer $a$ is called a congruent number if and only if there are positive integer solutions to the system of equations

$$
x^{2}+a y^{2}=z^{2} \text { and } x^{2}-a y^{2}=t^{2} .
$$

In this note congruent numbers are discussed and a table of known square-free congruent numbers less than 1000 is exhibited.
\end{abstract}

1. Introduction. An integer $a$ is called a congruent number if and only if there exist positive integer solutions to the rationalized system of Diophantine equations

$$
x^{2}+a y^{2}=z^{2} \text { and } x^{2}-a y^{2}=t^{2} .
$$

It is easy to see that $c n^{2}$ is a congruent number if and only if $c$ is a congruent number. Thus it suffices to study only square-free congruent numbers.

The earliest reference to congruent numbers in the literature dates back to a tenth century Arab manuscript. For a more detailed account of the early history of congruent numbers, see Dickson [3, Chapter 16, pp. 459-472]. More recent references to congruent numbers can be found in several texts. See, for example, Uspensky and Heaslet [6] and Mordell [5]. For the most recent results on congruent numbers, see Alter, Curtz and Kubota [1].

It is known that solving Eq. (1) is equivalent to solving the single Diophantine equation

$$
x^{4}-a^{2} y^{4}=z^{2}
$$

Also, every congruent number $a$ satisfying Eq. (1) must be of the form

$$
u v\left(u^{2}-v^{2}\right)=a w^{2} .
$$

There are other forms that a congruent number may take; in fact, the following are all congruent numbers:

$$
x^{4}+4 y^{4}, \quad 2 x^{4}+2 y^{4}, \quad x^{4}-y^{4} .
$$

If $x$ and $y$ have opposite parity, then the following are also congruent numbers:

$$
x^{4}+6 x^{2} y^{2}+y^{4}, \quad x^{4}-6 x^{2} y^{2}+y^{4} .
$$

In Alter, Curtz and Kubota [1], other results on congruent numbers and a list of known results on noncongruent numbers is given.

In 1915, Gérardin [4] listed 62 square-free congruent numbers less than 1000 for which $x$ is less than 3722 in Eq. (1). (The list actually contains 63 numbers but one is

Received January 26, 1973.

AMS (MOS) subject classifications (1970). Primary 65A05; Secondary $10 \mathrm{~B} 05$.

Key words and phrases. Congruent numbers, Diophantine equation.

Copyright (c) 1974, American Mathematical Society 
not square-free and thus is an error.) At the time he posed the problem of finding all square-free congruent numbers less than 1000 . This was followed by a note of Bastien [2] in which he listed all square-free congruent numbers less than 100 . Using new results and a computer search based primarily on Eq. (3), Alter, Curtz and Kubota [1] determine 334 of the 608 square-free numbers less than 1000 to be either congruent (198 numbers) or noncongruent (136 numbers). These numbers are exhibited in tables of congruent and noncongruent numbers.

It is the aim of this note to find new congruent numbers less than 1000 . This is done by using a computer search based on (4) and (5), in which the parameters were taken as large as double precision would allow. A discussion of this program can be found in [1]. In fact only 18 new congruent numbers were found, leaving 256 square-free numbers less than 1000 that are still undetermined.

A complete list of known (square-free) congruent numbers less than 1000 is contained below in Table 1 .

TABLE 1

Square-Free Congruent Numbers $<1000$

\begin{tabular}{rrrrrrrrrr|}
\hline 5 & 6 & 7 & 13 & 14 & 15 & 21 & 22 & 23 & 29 \\
30 & 31 & 34 & 37 & 38 & 39 & 41 & 46 & 47 & 53 \\
55 & 61 & 62 & 65 & 69 & 70 & 71 & 77 & 78 & 79 \\
85 & 86 & 87 & 93 & 94 & 95 & 101 & 102 & 109 & 110 \\
111 & 118 & 119 & 134 & 137 & 138 & 141 & 142 & 143 & 145 \\
149 & 151 & 154 & 158 & 159 & 161 & 165 & 166 & 174 & 181 \\
182 & 190 & 194 & 205 & 206 & 210 & 214 & 215 & 219 & 221 \\
226 & 231 & 239 & 246 & 254 & 255 & 257 & 262 & 265 & 278 \\
285 & 286 & 287 & 291 & 299 & 302 & 310 & 313 & 318 & 319 \\
323 & 326 & 330 & 334 & 349 & 353 & 357 & 358 & 366 & 371 \\
382 & 386 & 390 & 391 & 395 & 398 & 399 & 410 & 422 & 426 \\
429 & 434 & 438 & 442 & 445 & 446 & 454 & 455 & 457 & 462 \\
465 & 470 & 478 & 479 & 502 & 505 & 509 & 510 & 511 & 514 \\
517 & 518 & 526 & 527 & 533 & 535 & 542 & 546 & 561 & 565 \\
566 & 574 & 582 & 583 & 602 & 609 & 614 & 615 & 622 & 629 \\
645 & 646 & 651 & 658 & 662 & 663 & 669 & 670 & 671 & 674 \\
689 & 694 & 709 & 710 & 718 & 719 & 721 & 731 & 734 & 741 \\
751 & 758 & 759 & 761 & 766 & 777 & 791 & 793 & 798 & 799 \\
805 & 806 & 813 & 814 & 821 & 838 & 862 & 866 & 870 & 878 \\
879 & 886 & 889 & 890 & 897 & 901 & 903 & 905 & 910 & 915 \\
926 & 934 & 935 & 943 & 949 & 951 & 957 & 958 & 959 & 966 \\
974 & 982 & 985 & 987 & 995 & 998 & & & & \\
\hline
\end{tabular}

These new congruent numbers further strengthen the following conjecture which also appears in [1].

Conjecture. If $n \equiv 5,6$ or $7(\bmod 8)$ then $n$ is a congruent number.

The first three such $n$ still unsettled are 103, 127, 133 .

Although this note deals primarily with congruent numbers, our previously published list of known noncongruent numbers is repeated here for the convenience of the reader. For more information about Table 2, the reader is again referred to [1]. 
TABLE 2

Noncongruent Numbers $<1000$

\begin{tabular}{|rrrrrrrrrr|}
\hline 1 & 2 & 3 & 10 & 11 & 17 & 19 & 26 & 33 & 35 \\
42 & 43 & 51 & 57 & 58 & 59 & 66 & 67 & 73 & 74 \\
82 & 83 & 89 & 91 & 97 & 106 & 107 & 114 & 122 & 129 \\
130 & 131 & 139 & 146 & 163 & 170 & 177 & 178 & 179 & 186 \\
193 & 201 & 202 & 209 & 211 & 218 & 227 & 233 & 241 & 249 \\
251 & 274 & 281 & 283 & 290 & 298 & 307 & 314 & 321 & 331 \\
346 & 347 & 362 & 370 & 379 & 393 & 394 & 401 & 417 & 419 \\
433 & 443 & 449 & 457 & 458 & 466 & 467 & 473 & 489 & 491 \\
499 & 523 & 530 & 537 & 538 & 547 & 554 & 562 & 563 & 571 \\
586 & 587 & 601 & 610 & 617 & 619 & 626 & 633 & 634 & 641 \\
643 & 649 & 659 & 673 & 681 & 683 & 691 & 698 & 737 & 739 \\
746 & 753 & 754 & 769 & 778 & 787 & 794 & 811 & 817 & 818 \\
827 & 842 & 849 & 859 & 883 & 907 & 913 & 914 & 921 & 922 \\
929 & 937 & 947 & 962 & 971 & 993 & & & & \\
\hline
\end{tabular}

The author would like to thank $\mathbf{R}$. Waldner for programming assistance.

Department of Computer Science

University of Kentucky

Lexington, Kentucky 40506

1. R. Alter, T. B. Curtz \& K. K. Kubota, Remarks and Results on Congruent Numbers, Proc. Third Southeastern Conference on Combinatorics, Graph Theory and Computing, 1972, pp. 27-35.

2. L. BASTIEN, "Nombres congruents," Intermédiare des Math., v. 22, 1915, pp. 231-232.

3. L. E. Dickson, History of the Theory of Numbers. Vol. II, Carnegie Institute of Washington, 1920.

4. A. GÉRARDIN, "Nombres congruents," Intermédiare des Math., v. 22, 1915, pp. 52-53.

5. L. J. MORDELl, Diophantine Equations, Pure and Appl. Math., vol. 30, Academic Press, New York, 1969. MR 40 \#2600.

6. J. V. USPENSKY \& M. A. HeAsLet, Elementary Number Theory, McGraw-Hill, New York, 1939. MR 1, 38. 